\title{
CRITIQUE DE LA VIOLENCE. WALTER BENJAMIN ET L'IDÉE DE « DESTITUTION DU DROIT »
}

\author{
TOBIAS NIKOLAUS KLASS
}

\begin{abstract}
The article proposes an analysis of Walter Benjamin's writings on law, more specifically on the author's critique of the violent dimension of law. It seeks to analyze the genesis of the concept of "dispossession of law" ["Entsetzung des Rechts"] and its use within Benjamin's writings. The article is divided into three parts: the first part seeks to contextualize Benjamin's critique of law in light of the Marxian conception of right. In a second moment, the notion of "Entsetzung des Rechts" is analyzed, as well as its possible interpretations based on the semantic scope inscribed in the term and in the German language. Finally, the paper will seek to clarify the notion of "Entsetzung des Rechts" in relation to other concepts and texts of the author.
\end{abstract}

\section{Introduction}

L'objectif de mon exposé sera en quelque sorte un objectif très modeste : il s'agit de comprendre la réponse qu’a donnée Walter Benjamin à sa propre critique de la violence, qui est d'abord une critique de la violence du droit. La réponse de Benjamin se résume dans sa formule destitution du droit qui donne, en allemand : Entsetzung des Rechts (je cite la formule en allemand parce qu'il sera indispensable pour mes réflexions de comprendre la composition du mot Entsetzung afin de comprendre ce que Benjamin voulait dire par là). ${ }^{1}$

1 Les écrits de Benjamin sont cités d'après l'édition allemande : Gesammelte Schriften (GS), t. I-VII, Suhrkamp Verlag, Francfort-sur-le-Main 1974-1989; pour les textes qui ont été traduits en français je me réfère aux Euvres, t. I-III, Gallimard, Paris 2000.

https://doi.org/10.14712/24646504.2021.3

(C) 2021 The Author. This is an open-access article distributed under the terms of the

Creative Commons Attribution License (http://creativecommons.org/licenses/by/4.0). 
Pour élucider la formule Entsetzung des Rechts je procèderai en trois étapes. Dans un premier temps je rappellerai très brièvement et de manière fortement abrégée le contexte historique et théorique, pour et dans lequel Benjamin a forgé la formule. Puis, je regarderai de plus près la formule elle-même, c'est-à-dire les possibilités sémantiques qu'elle contient et avec lesquelles elle joue. Ces possibilités sémantiques sont d'un côté les possibilités qu'on trouve dans le texte Critique de la violence lui-même ; de l'autre côté elles renvoient à l'œuvre de Benjamin dans sa totalité, c'est-à-dire elles visent un au-delà du texte en question (en restant tout de même des possibilités benjaminiennes). Cette deuxième étape mettra en lumière deux interprétations différentes de la formule Entsetzung des Rechts. Pour conclure, j'essaierai dans un troisième temps de mieux comprendre une de ces deux interprétations - à savoir l'interprétation qui est moins connue, mais qui est, à mon avis, l'interprétation plus féconde à long terme - en donnant un exemple concret illustrant la valeur pratique possible de cette interprétation.

\section{1.}

Comme je le disais au début, la Critique de la violence de Benjamin est d'abord et surtout une critique de la violence du droit. Toutefois, parler d'une violence $d u$ droit peut - en français - mener à une distorsion du sens original ; le mot utilisé par Benjamin dans ce contexte est le mot allemand Gewalt et, contrairement au mot francophone "violence ", le mot germanophone Gewalt n'est pas pris d'abord comme une violence physique ou corporelle. En allemand on appelle, par exemple, la séparation des pouvoirs, Gewaltenteilung, l'autorité de l'état se veut chez nous comme Staatsgewalt et la force publique est appelée öffentliche Gewalt. C'est-à-dire, au terme allemand Gewalt on associe des phénomènes qui sont en français rendus comme force, pouvoir ou même puissance ; des formes plutôt structurelles et abstraites de violence, qui ne sont pas a priori physiques ou corporelles. Comme, par exemple, le droit.

Dans sa critique de la violence (Gewalt) du droit, Benjamin poursuit, bien entendu, la tradition marxiste de la critique du droit, mais il donne une forme très singulière à cette tradition critique. Pour Marx le point crucial est, grosso modo, qu'il reproche au droit de prendre position pour ceux qui possèdent (surtout les moyens de production), les propriétaires, contre ceux qui n'ont rien, les démunis. La critique du droit ou de la violence du droit benjaminienne n'est, par contre, pas simplement une prise de position contre les propriétaires et pour les démunis (même si une prise de position pour les vaincus de l'histoire joue un 
rôle important dans la pensée de Benjamin). ${ }^{2}$ Mais chez Benjamin la critique du droit concerne plutôt la violence structurelle du droit même - d'un droit qui reçoit toute sa légitimité de l'affirmation de mettre fin à toute violence (par exemple, de la violence de l'état de nature). Cette violence spécifique du droit, Benjamin la localise d'abord dans la violence de ses moyens. Le point de départ de la critique des moyens du droit (des Rechtsmittel) est la détermination des moyens par les fins. Le droit, c'est ça le nœud de la critique fondamentale du droit de Benjamin, n'est pas capable de voir la violence de ces moyens, parce qu'il est incapable de regarder les moyens comme moyens seulement, en tant que tels, hors de chaque détermination par les fins. Parce que chaque violence des moyens est toujours justifiée à l'avance par les fins (soit des fins justes soit des fins injustes).

Et ce n'est pas tout. En outre, Benjamin montre - et c'est ici que sa critique prend sa forme spécifique - que la détermination des moyens comme moyens pour des fins est imprégnée d'une hypocrisie profonde. En effet, chaque droit prétend être violent juste en cas d'urgence, c'est-à-dire dans le cas où le droit tout court est menacé de l'extérieur dans son existence; dans ce cas-là, le droit a non seulement le droit mais le devoir de se défendre contre l'agression extérieur pour protéger l'effet pacifiant du droit en général. Cette fonction du droit, Benjamin l'appelle conservatrice de droit (en allemand : rechtserhaltend). ${ }^{3}$ Pourtant Benjamin nous dit que cette autodescription est une auto-description trompeuse (et en cela sa critique se révèle être une sorte de critique de l'idéologie au sens marxiste du terme). Pourquoi une telle auto-description est-elle trompeuse ? Parce que chaque violence de droit ne sert pas simplement à la conservation du droit, mais elle est toujours et en même temps fondatrice de droit (rechtssetzend). ${ }^{4}$ Autrement dit : chaque violence de droit ne défend pas simplement les frontières qui ont été tracées pour protéger un espace clos et dont la transgression menace de détruire l'espace qu'elles protègent, mais la violence de droit fonde aussi du droit, elle est rechts-setzend (setzen dans ce cas ne veut pas simplement dire fonder, mais aussi mettre en place, fixer ou même déterminer). Avec cela, la violence de droit ne conserve pas simplement du droit déjà constitué, mais elle constitue elle-même du droit. Elle est fondatrice de droit.

Pour illustrer cette double force du droit, Benjamin attire notre attention sur la violence exercée par la police. A première vue et selon l'image qu'elle se fait d'elle même (qui est en même temps le fond de sa légitimation) la violence de la police

2 Voir p. ex. : Benjamin, Walter : Über den Begriff der Geschichte, GS I (2), p. 691-704; Sur le concept de l'histoire, Euvres, t. III, pp. 427-443.

3 Voir : Benjamin: Zur Kritik der Gewalt, GS II (1), pp. 179-203 ; p. 187 ; Euvres, t. I, p. 210-243 ; p. 220.

4 Benjamin : Zur Kritik..., op. cit., p. 186 ; Benjamin : Euvres..., op. cit., p. 220. 
sert d'abord à défendre le droit et ses frontières. Dans un État de droit il est possible de manifester dans les rues, c'est un droit protégé et garantit par la police et sa violence; mais au moment où les manifestants abusent de ce droit à manifester pour des fins qui mettent en question le droit en tant que tel, la police, elle, a le droit et même le devoir - pour protéger le droit tout court - de s'attaquer aux manifestants si nécessaire par la force. Une telle argumentation semble à première vue convaincante ; mais Benjamin fait apparaître son caractère intrinsèquement partiel. Pourquoi ? Parce que la décision de savoir si une manifestation est encore une manifestation conforme au droit ou si elle a déjà transgressé les frontières de la légalité, cette décision ne se laisse pas déduire du droit même, mais elle est - au sens de Carl Schmitt - une décision au sens primordial. Car elle doit être prise à chaque fois à nouveau dans la situation donnée selon les convictions et le jugement des policiers sur place, dont la tâche est de déterminer d'abord les frontières qui ont, selon eux, été transgressées par les manifestants. C'est ici que se manifeste quelque chose de corrompu au cour du droit (etwas Morsches im Recht). ${ }^{5}$ Le droit justifie sa propre violence en renvoyant à la nécessité de se conserver contre les attaques extérieures afin de protéger les citoyens contre la violence : ce n'est en réalité qu'un prétexte pour cacher qu'il se protège d'abord lui-même, c'est-à-dire son pouvoir, dont il craint la perte. Ce moment décisioniste qu'on n'arrive jamais à empêcher ou éviter complètement, signifie que chaque application du droit n'est jamais juste un acte de conservation, mais aussi de fondation, d'institution du droit. Or, le droit n'existe que dans de telles applications, il n'est que enforced (comme Derrida l'a souligné dans sa lecture du texte de Benjamin). ${ }^{6}$

C'est précisément ce moment constitutif cette force d'instituer du droit au moment de sa défense toujours au moins virtuellement présent dans chaque acte judiciaire que Benjamin met en cause fondamentalement sous le titre critique de la violence. C'est aussi ici qu'entre en jeu l'idée de la destitution du droit comme réponse au problème de la violence instituante du droit. Que signifie destitution du droit?

\section{2.}

Avec cela j’ai terminé la première partie de mon exposé - reconstruction schématique du contexte théorique, pour et dans lequel Benjamin a forgé la formule des-

5 Benjamin : Zur Kritik..., op. cit., p. 188 ; Benjamin : Euvres..., op. cit., p. 223.

6 Cf. Derrida, Jacques: Force de loi, Galilée, Paris 1994. 
titution du droit - et je passe à la deuxième partie : l'interprétation de la formule elle-même. À première vue la formule - dans sa version allemande Entsetzung des Rechts - est due à la possibilité de la langue allemande de former des composés. Le mot central Entsetzung - qui n'existe pas comme tel dans le langage courant - est évidemment un mot artificiel : le mot est composé du préfixe Ent- - qui annonce une négation, un acte d'interruption ou d'arrêt - et le mot Setzung, qui signifie exactement le nœud de la critique de Benjamin de la violence du droit : sa puissance d'instituer ou de fonder un droit sans raison et comme simple acte du pouvoir. Ent-Setzung comme telle est l'expression d'une négation, plus précisément l'expression de l'idée d'interrompre ou d'arrêter la puissance d'institution ou de fondement des actes juridiques.

Cette idée - qui est sur le plan méthodologique celle d'une négation déterminée au sens hégélien du terme ; de ce côté Benjamin est très proche d'Adorno - est illustrée par Benjamin par son interprétation du mythe de Niobé (dans Critique de la violence). Niobé, l'épouse d'Amphion, roi de Thèbes, a eu sept fils et filles, ce qui est beaucoup plus d'enfants que le titan Leto avec ses deux enfants Artémis et Apollon. Évidemment il n'y avait pas de loi interdisant à un être humain d'avoir plus d'enfants qu'un titan ; or, Niobé n'a pas transgressé la loi, elle n'est pas passible d'une sanction juridique. Néanmoins, Artémis et Apollon tuèrent tous les enfants de Niobé pour établir une frontière entre les hommes et les dieux, et pour soumettre Niobé à l'expiation (der Sühne überantworten). ${ }^{7}$ Ce trait de la violence mythique - dont le principe est le pouvoir et justement pas la justice - est en même temps le nœud de la violence du droit, comme en témoigne selon Benjamin « le principe moderne selon lequel l'ignorance de la loi ne garantit point du châtiment $»{ }^{8}$ Niobé, avec tous ses enfants, a transgressé une frontière dont elle ignorait l'existence ; elle n'en a pas moins été punie, c'est-à-dire elle a été soumise à l'expiation. Ce qu'on peut avec raison considérer comme injuste parce que Niobé non seulement ignorait l'existence de ladite frontière, mais elle ne pouvait pas la connaître, parce que ladite frontière a été établie, institué (gesetzt) à proprement parler juste au moment de l'acte puissant et violent d'Artémis et d'Apollon - tout comme, selon Benjamin, la police établit des frontières juste au moment où elle décide que les manifestants ont transgressé la même frontière. Dans ce sens la violence de droit se révèle comme identique à la violence mythique, parce qu'elle aussi « fonde un droit bien plutôt qu'elle ne punit une infraction à un droit existant ». ${ }^{9}$

\footnotetext{
Benjamin : Zur Kritik..., op. cit., p. 200 ; Benjamin : CEuvres..., op. cit., p. 237.

Benjamin : Zur Kritik..., op. cit., p. 199; Benjamin : Euvres..., op. cit., p. 237.

Benjamin : Zur Kritik..., op. cit., p. 197 ; Benjamin : Euvres..., op. cit., p. 234.
} 
A cette injustice du pouvoir du droit de fonder ou d'instituer soi-même des frontières (au lieu de les conserver seulement) afin de garder son pouvoir (comme le fait pouvoir mythique), Benjamin, lui, oppose un moyen qu'il appelle «moyen pur». Un tel moyen est un moyen libéré de son attachement à des fins : il est alors à comprendre comme un intermédiaire entre des sphères bien séparées, plutôt que comme un moyen au sens instrumental (le mot allemand Mittel signifie moyen mais en même temps intermédiaire). Ce moyen pur caractériserait la violence divine. La tâche de la violence divine doit d'abord s'opérer par une négation déterminée : "Si la violence mythique, écrit-il, est fondatrice de droit (rechtssetzend), la violence divine est destructrice de droit (rechtsvernichtend); si l'une pose des frontières (setzt [...] Grenzen), l'autre est destructrice sans limites (vernichtet diese grenzenlos); si la violence mythique impose tout ensemble la faute et l'expiation (verschuldend und sühnend), la violence divine lave de la faute (ist ent-sühnend) $» .{ }^{10}$ Destitution du droit (Ent-Setzung des Rechts) comme tâche première et primordiale du moyen pur nommé violence divine est, par conséquence, d'abord un processus de négation pas à pas : ce qui a été soumis à l'expiation (Sühne) en est libéré (ent-sühnt), ce qui a été rendu coupable pour avoir transgressé une frontière est libéré de sa faute par la destruction de toutes les frontières. Destitution dans le sens de Ent-Setzung signifie alors la négation du pouvoir d'instituer, de la Setzungs-Macht du droit, négation qui s'opère par la destruction des frontières qui ont justement produit la faute et l'injustice. Or, la réponse à l'injustice (et avec cela à la violence) du droit est, semble-t-il, la destruction du droit même (Rechtsvernichtung) et en même temps - c'est la formule de la violence divine qui nous renvoie à cette idée - elle induit de se pencher vers un au-delà du droit (Jenseits des Rechts) que Benjamin évoque dans les dernières phrases de son texte. Avec cela nous sommes arrivés au cour de la théologie négative de l'acte révolutionnaire, dont la tâche est de mettre en œuvre la destitution du droit : un acte de violence divine sécularisé signant la destruction complète du système juridique.

L'interprétation du mot artificiel Entsetzung des Rechts présentée jusqu'ici est une interprétation qui peut sans doute réclamer une certaine force de persuasion, mais elle n'est pas la seule possible. Dans le texte Critique de la violence, il y a déjà quelques indications visant à d'autres interprétations. Ceux qui connaissent un peu le texte savent que Benjamin s'oppose, par exemple, à un anarchisme naif, qui ferait résider le bonheur dans la destruction de tout ordre (la critique claire de l'anarchisme tout bonnement puéril qu'on trouve dans le texte ne laisse pas des doutes

10 Benjamin : Zur Kritik..., op. cit., p. 199 ; Benjamin : Euvres..., op. cit., p. 238. 
sur ce sujet). ${ }^{11}$ Le droit est pour Benjamin un état de violence grâce à sa puissance ou son pouvoir de poser arbitrairement des frontières (Setzungen), pouvoir qu'il faut critiquer et abroger; mais : le résultat de cette critique ne peut pas mener à un état de désordre absolu. Au contraire : là, où Benjamin explique - toujours dans le texte Critique de la violence - le passage des moyens du droit, qui sont toujours des moyens visant à des fins, à ce qu'il appelle des moyens purs, il montre que ces derniers sont déterminés par la loi (durch das Gesetz). ${ }^{12}$ Alors que jusqu'ici Benjamin n'a pas fait de différence entre les termes de droit et de loi, il sépare clairement à cette occasion la loi évoquée ici du droit; à proprement parler, il y oppose le droit positif (faits des êtres humains) à une loi qui est une loi divine plus ou moins inaccessible aux hommes (ce qui renvoie une fois de plus à des traits théologiques dans la pensée de Benjamin). Mise à part la métaphysique de la loi divine, la figure de la loi divine montre bien que pour Benjamin ce ne peut être qu'une forme nommée loi (Gesetz) qui peut mener à la justice et non l'absence absolue de règles. Aux contraintes du droit qui est une forme de violence Benjamin n'oppose pas la formule tout ce qui plait est permis, mais une forme spécifique de contrainte qui ne porte pas en soi la violence de la contrainte juridique. Ce n'est pas pour rien que l'alternative à la violence de droit n'est pas d'abord et uniquement la non-violence, mais une forme de violence que Benjamin appelle violence divine.

Ces quelques réflexions brèves et superficielles ont, j'espère, suffi à rendre évident qu'il est possible de poser autrement la question Qu'est-ce qu'est l' 'Entsetzung des Rechts'? Pour ce faire, je reviens encore une fois au mot artificiel Ent-Setzung, c'est-à-dire à ses composés et à sa composition. Car le mot allemand Entsetzung est non seulement une négation (Ent-) de l'idée de l'institution ou de la constitution (Setzung), mais on peut y entendre facilement une allusion au verbe entsetzen, dont la forme nominale est le nom Entsetzen. La signification de Entsetzen varie selon le contexte et renvoie soit à l'horreur ou à la peur, soit á l'épouvante ou même à la terreur. Benjamin lui-même utilise l'idée d'un Entsetzen à plusieurs reprises dans ses textes les plus célèbres - comme, par exemple, dans les essais sur l'œuvre de Baudelaire, où l'Entsetzen prends la forme du choc, mais aussi dans les thèses sur l'histoire, où Benjamin voit un Entsetzen dans le visage de l'ange de l'histoire de Paul Klee face à la catastrophe qui est l'histoire : "Ses yeux sont écarquillés, sa bouche ouverte, ses ailes déployées ».13

Qu'est-ce qu'un tel Entsetzen au sens de choc, d'horreur, a avoir avec le Entsetzung dans la formule Entsetzung des Rechts? Pour répondre à cette question,

11 Benjamin : Zur Kritik..., op. cit., p. 187 ; Benjamin : CEuvres..., op. cit., p. 220.

12 Benjamin :Zur Kritik..., op. cit., p. 191 ; Benjamin : Euvres..., op. cit., p. 227.

13 Benjamin : Über den Begriff..., op.cit.,p. 697; Sur le concept..., op.cit. p. 434. 
permettez-moi d'attirer votre attention sur un texte moins connu de Benjamin, publié la première fois en 1929 dans les Denkbilder (images de pensée), qui, à mon avis, illustre le mieux le nœud de l'idée d'Entsetzen dans la Critique de la violence. Le texte porte le titre Schönes Entsetzen (Belle horreur). Malheureusement il n'y a pas de traduction française de ce texte et je n'ose pas le traduire, comme son langage est assez dense et complexe. Je me contente donc de présenter la version allemande suivie d'un résumé des traits les plus importants en français :

Der vierzehnte Juli. Von Sacré-Cœur aus übergießen bengalische Feuer Montmartre. Der Horizont hinter der Seine glüht. Feuergarben fahren auf und erlöschen über der Ebene. Zehntausende stehen am steilen Abhang gedrängt und folgen dem Schauspiel. Und diese Menge kräuselt unaufhörlich ein Flüstern wie Fältchen, wenn der Wind im Mantel spielt. ${ }^{14}$

Dans la première partie du texte - j'ai divisé le texte en trois parties; plus tard vous verrez pourquoi - Benjamin décrit les festivités du 14 juillet à Paris. Il y a un feu d'artifice impressionnant et une foule suivant le spectacle.

Spannt man sein Ohr dem schärfer entgegen, so tönt darin noch anderes als Erwartung der Raketen und Leuchtkugeln. Erwartet nicht diese dumpfe Menge ein Unheil, groß genug, aus ihrer Spannung den Funken zu schlagen; Feuersbrunst oder Weltende, irgend etwas, das dies samtne, tausendstimmige Flüstern umschlagen ließe in einen einzigen Schrei, wie ein Windstoß das Scharlachfutter des Mantels aufdeckt? Denn der helle Schrei des Entsetzens, der panische Schrecken ist die Kehrseite aller wirklichen Massenfeste. Der leise Schauer, der die ungezählten Schultern überrieselt, bangt nach ihm. Für das tiefste, unbewußte Dasein der Masse sind Freudenfeste und Feuersbrünste nur Spiel, an dem sie auf den Augenblick des Mündigwerdens sich vorbereitet, auf die Stunde, da Panik und Fest, nach langer Brudertrennung sich erkennend, im revolutionären Aufstand einander umarmen.

La deuxième partie signifie une rupture presque imperceptible (il faut, pour la percevoir, aiguiser son oreille, die Ohren schärfen), mais d'une grande importance avec la scène originale : Benjamin entrevoit quelque chose derrière la fascination des masses pour la beauté du feu d'artifice, quelque chose venant de l'être inconscient de la masse (das tiefste, unbewusste Dasein der Masse) : des attentes de quelque chose d'imprévu, et en même temps catastrophique (un Unheil, malheur, la fin du monde: Weltende; comme dans son texte sur Baudelaire Benjamin se

14 Benjamin : GS IV (2), p. 434. 
réfère à la théorie de l'angoisse d'Au-delà du principe de plaisir de Freud ${ }^{15}$ ) qui tournerait la fascination enfantine en un cri d'horreur (Schrei des Entsetzens), qui est en même temps l'expression de l'ivresse de la fête (l'ivresse de la fraternité, une idée empruntée à Bloch) et d'une panique face à l'horreur. Selon Benjamin, ces deux - l'ivresse fraternelle de la fête et la paniques face à l'horreur - se serrent dans les bras (die einander umarmen) au moment de l'insurrection révolutionnaire (revolutionären Aufstand). Ce moment révolutionnaire signifie, dit Benjamin, le moment de l'émancipation (Mündigwerden) de la masse.

Jusqu'ici tout semble bien connu : c'est le moment d'un choc des masses en ivresse (presque dionysiaque) produit par une interruption de l'ordre établi par l'événement violent de l'insurrection révolutionnaire (la forme sécularisée de la violence divine) qui est le moment de l'émancipation des masses.

Mais Benjamin ne s'arrête pas ici, vient s'ajouter une troisième partie qui consiste en une seule phrase : "Von Rechts wegen begeht man in Frankreich die Nacht des vierzehnten Juli mit Feuerwerk ». Aux festivités du 14 juillet il y a toujours dans la nuit un feu d'artifice - à savoir von Rechts wegen, prescrit par le droit, qui est de droit.

Or, ce que nous trouvons dans le texte Schönes Entsetzen c'est la même configuration théorique que dans la Critique de la violence (et dans d'autres textes célèbres de Benjamin de cette période), sauf que dans ce petit texte toute la configuration ou constellation est elle-même une mise en scène théâtrale par le droit même - von Rechts wegen. Nous y trouvons la figure d'un au-delà du droit dans le droit même, dont Derrida a parlé dans son texte Le siècle et le pardon (sans malheureusement préciser cette idée) ${ }^{16}$ : la figuration d'un endroit ou d'un lieu de non-droit dans le droit même. Ent-setzen des Rechts n'y est plus l'abolition totale du droit par un acte révolutionnaire, mais le moment de l'Entsetzen est une sorte d'interruption théâtrale, un épochè instantané, qui a la force de produire un temps mort, de clôturer le moment pour quelques instants seulement par un mur qui sert à protéger l'espace constitué contre le droit, ou plus précisément : contre les forces du droit, contre sa violence, sa Setzungsmacht, sa violence d'institution. Comme ça, l'Entsetzen des Rechts deviendrait ce que Benjamin lui-même appelle la délivrance de l'emprise exercée (die Erlösung aus dem Bannkreis) par toutes les formes du droit : la rédemption de l'envoutement du droit, de ses forces de contrainte. En constituant dans le droit même un lieu où le droit comme puissance est impuissant, parce que ses forces sont mises entre parenthèses; comme le silence au centre de l'ouragan.

15 Voir Benjamin: Über einige Motive bei Baudelaire, GS I (2), pp. 605-653 ; p. 612 ff.

16 Voir Derrida: Le Siècle et le Pardon, dans : Foi et savoir, Seuil, Paris, 2001, pp. 101-133. 


\section{3.}

La question maintenant est, évidemment, comment s'imaginer concrètement ce lieu de non-droit dans le droit? Penser l'Entsetzung des Rechts comme destruction complète de chaque système juridique par un acte de violence révolutionnaire sans savoir ce qui se passe après - cela nous connaissons tous bien, trop bien peut-être ; l'attitude révolutionnaire risque, à mon avis, de trop aisément ignorer toute la souffrance qu'elle produit. Or, comment penser dans la réalité ou la vie quotidienne la deuxième interprétation de l'Entsetzung des Rechts comme au-delà du droit dans le droit même ? Est-ce que ce n'est pas une pure théorie qui peut être - éventuellement - convaincante pour la lecture du texte benjaminien, mais qui est sans la moindre valeur pratique?

Ce n'est pas, je crois, sans raison que Jacques Derrida s'est penché sur l'idée d'un au-delà du droit dans le droit même dans le contexte du pardon, plus précisément du pardon politique. A première vue cette idée semble une idée impossible. Le pardon est, de toute évidence, un acte social limité à deux, l'ingérence d'un tiers ne peut que pervertir l'acte : personne ne peut pardonner pour quelqu'un d'autre et personne ne peut demander pardon pour quelqu'un d'autre. Si cela a lieu quand même, il ne peut s'agir que d'une instrumentalisation politique d'un acte en soi apolitique - au détriment surtout des victimes (comme Sandrine Lefranc l'a illustré de manière très convaincante par l'exemple des commissions de vérité ou de réconciliation en Afrique du Sud après la fin du système d'apartheid). ${ }^{17}$

Je crois, pourtant, qu'il est sensé de demander s'il n'y a pas un lien entre l'idée d'une Entsetzung des Rechts et celle du pardon. Une première indication pour un tel lien est donnée par le concept d'expiation (Sühne) et sa négation (Ent-Sühnung). Encore une fois : le pardon a nécessairement lieu entre deux personnes agissantes ; personne ne peut pardonner à la place de la victime (moi, je ne peux pas pardonner à toi ce que tu as fait à un tiers); et personne ne peut demander pardon à la place du coupable. C'est pour cela que l'autre du pardon est la vengeance et jamais la punition : car la punition est toujours l'affaire d'un tiers (normalement : le juge) qui applique une sanction qui transgresse les deux (car il vaut pour chacun). Par contre, la vengeance est toujours l'affaire des parties au conflit elles-mêmes : elle a lieu entre celui qui a subi un dommage, une blessure, etc., et celui qui est à l'origine de ce dommage, blessure, etc. La vengeance comme acte a pour objectif de remplacer l'acte initial par un deuxième acte (un contre-acte) qui a la force de compenser la faute initiale ; la vengeance est une question d'équilibre perturbé et

17 Voir : Lefranc, Sandrine : Politiques du Pardon, Presses Universitaires de France, Paris 2002. 
ensuite restitué. Il est évident que la faute, qui est affaire des deux parties et qui ne peut être traitée par un tiers, ne peut pas être compensée par une peine (au sens juridique), mais par la vengeance seulement - ou par l'Ent-sühnung, c'est-à-dire la négation de l'" expiation » : le "laver de la faute». Parce que ce que fait l'Entsühnung c'est annuler la dette de l'autre - au lieu de la compenser par une contre-faute.

Dans le texte Critique de la violence dans lequel l'expiation et sa négation (son annulation) jouent un rôle si important, Benjamin ne parle pas du pardon. Mais il en parle dans un fragment de 1921 (publié dans les fragments posthumes) ${ }^{18}$, intitulé La signification du temps pour le monde morale (Die Bedeutung der Zeit für die moralische Welt). ${ }^{19}$ Ce texte nous aide à mettre en relation l'idée d'une expiation niée, annulée, et le pardon (au moins structurellement). Comme il n'y a pas de traduction de ce texte dense et complexe, j'en donne - encore une fois - un résumé fortement abrégé et ne regarde de près que les passages les plus importants pour notre question.

Le point du départ du texte en question est la prétendue intemporalité du droit, qui garantit communément sa moralité. L'idée en est simple : le droit doit être juste indépendamment du temps et de l'espace (cultuelle). Celui qui est jugé coupable par le droit (ou jugé avoir commis un acte criminel) doit recevoir la même sanction hier ou demain, ici ou là, parce que la justice appliquée ou mise en œuvre par le droit est comprise comme étant universelle. L'objectif du fragment en question est de contredire fondamentalement cette idée : pour Benjamin c'est justement l'intemporalité qui distingue le droit de la moralité (ou, plus généralement, de l'idée de justice). Pourquoi cela ? Parce que, dit Benjamin, le droit suit dans son intemporalité l'idée de la vengeance : pour laquelle une faute n'est expiée qu'au moment où une contre-faute a compensé la faute première. Cette idée d'un équilibre perturbé par la faute originelle rééquilibré par une contre-faute, c'est ça l'idée de base de la vengeance; et pour Benjamin cela vaut également pour le droit. Nous connaissons cet argument de la Critique de la violence : où la vengeance d'Artémis et d'Apollon se révèlent comme identique au principe du droit, « selon lequel l'ignorance de la loi ne garantit point du châtiment ».20

Contrairement au droit - comme l'avance Benjamin dans la suite de son argumentation - le temps ou la temporalité jouent un rôle important pour la morale : car pour elle les choses - surtout les crimes - changent dans l'écoulement du temps. Ce qui ne signifie point pour lui que dans le temps on oublie tout ou que

18 Voir : Benjamin: Die Bedeutung der Zeit in der moralischen Welt, GS VI, p. 97-98.

19 Pour une interprétation instructive du fragment, voir : Rauen, Verena: Die Zeitlichkeit des Verzeihens. Zur Ethik der Urteilsenthaltung, Fink, Paderborn 2015.

20 Benjamin : Zur Kritik..., op. cit., p. 199 ; Benjamin : CEuvres..., op. cit., p. 237. 
le temps guérit tout. "La signification du temps, écrit Benjamin, ne se révèle pas au monde du droit où règne la vengeance, mais seulement là où dans le monde moral le pardon s'oppose à la vengeance » (« Die Bedeutung [der Zeit], erschießt sich nicht in der Welt des Rechts, wo die Vergeltung herrscht, sondern nur, wo ihr, in der moralischen Welt, die Vergebung entgegen tritt "). ${ }^{21}$ Pour expliquer cette affirmation Benjamin se réfère encore une fois à un concept mythique : $s$ 'inspirant de l'idée de l'Ancient Testament d'un délai de la faute qui sera arrêté par l'évènement du Jugement dernier (comparable en cela au jugement du droit qui met fin à la question de la faute au moment du jugement), Benjamin, lui, cherche à établir une autre idée du délai. Le temps du pardon, écrit-il, s'enfuit sans cesse dans l'avenir (unablässig ins Zukünftige flüchtend), de telle sorte que contre le silence solitaire de l'angoisse (die einsame Windstille der Angst) qui s'empare du criminel face au Jugement, se lève la tempête mugissante du pardon devant le Jugement qui s'approche (vorm immer nahenden Gericht daherbrausende laute Sturm der Vergebung). "Cette tempête, Benjamin conclut, n'est pas seulement la voix dans laquelle s'estompe le cri d'angoisse du criminel, mais elle est aussi la main qui élimine les traces de sa faute, même s'il lui faut dévaster pour cela la terre. " ("Dieser Sturm ist nicht nur die Stimme in der der Angstschrei des Verbrechers untergeht, er ist auch die Hand, welche die Spuren seiner [Untat] vertilgt, und wenn sie die Erde darum verwüsten müsste »). ${ }^{22}$

Le scénario présenté ici est imprégné des mêmes traits théologiques et de la même brutalité que la violence divine que nous connaissons du texte Critique de la violence: le pardon, initié par la colère de Dieu, dévaste la terre. Mais Benjamin ne serait pas Benjamin s'il ne cherchait en ce point barbare, en cet instant du pire, où la négation est la plus forte, le renversement dialectique. "Ce que cette image nous dit, conclut-il dans sa démonstration, il faut le saisir avec des concepts clairs et distincts : la signification du temps dans l'économie du monde morale, non seulement y efface les traces de la faute, mais y permet - par-delà tout souvenir ou tout oubli - le pardon par sa durée, bien que jamais la réconciliation » (" Was in diesem Bild gesagt ist, muss sich klar und deutlich in Begriffen fassen lassen: die Bedeutung der Zeit in der Ökonomie der moralischen Welt, in welcher sie nicht allein die Spuren der Untat auslöscht, sondern auch in ihrer Dauer - jenseits allen Gedenkens oder Vergessens - auf ganz geheimnisvolle Art zur Vergebung hilft, wenn auch nie zur Versöhnung »). ${ }^{23}$

\footnotetext{
Benjamin : Die Bedeutung..., op. cit., p. 97.

Ibid., p. 98.

Ibid.
} 
Il n'est pas facile de comprendre ce que Benjamin essaie de saisir dans ce fragment : l'idée d'un pardon, qui n'est ni simplement souvenir ni oubli, qui pourtant est capable d'effacer les traces de la faute du coupable, sans jamais déboucher sur la réconciliation (parce que seules les traces de la faute sont éliminées, non la faute même), en se transformant dans le délai permanent du Jugement (qu'on trouve, dans sa version négative, si souvent dans les textes de Kafka).

Qu'est-ce qu'apporte cette vision du pardon? Au moins ceci, il me semble : si la négation ou l'annulation de l'expiation (Ent-sühnung) est une forme possible de destitution du droit (Entsetzung des Rechts), et si le pardon présenté il y quelques instants est un moyen permettant d'annuler l'expiation (parce qu'il élimine les traces de la faute sans jamais conduire à la réconciliation), dans ce cas-là cette forme du pardon pourrait être un bon candidat pour l'idée d'un pardon engendrant une Entsetzung des Rechts hors de l'abolition complète du système juridique. À condition - bien entendu - qu'on réussisse à traduire cet au-delà-du-droit, qui est la moralité présentée ici, dans le droit même.

Est-ce que c'est possible ? Je pense que oui, même si je ne peux, pour conclure, que donner quelques indications concernant une telle possibilité. Structurellement il devrait consister en une sorte de droit non-souverain à pardonner. Ce droit non-souverain à pardonner serait l'exacte contre-image du droit souverain à pardonner : comme le droit de grâce du président. Ce droit souverain à pardonner qui est la grâce présidentielle est l'exception avec laquelle la souveraineté affirme sa puissance (au sens de Carl Schmitt : " est souverain celui qui décide l'état d'exception »). Mais ce droit souverain n'est pas le seul état d'exception du droit existant. De l'autre côté de l'échelle politique on trouve déjà aujourd'hui, par exemple, le droit de l'accusé de se taire, où le droit même accorde un droit de se retirer du droit dans le droit même. Le droit non-souverain à pardonner serait un tel « véritable état d'exception » (dont Benjamin a parlé dans la célèbre huitième thèse sur l'histoire ${ }^{24}$ ), c'est-à-dire un droit non pas des puissants mais des impuissants. Un tel droit ne serait pas un droit des coupables à demander pardon (qui sera nécessairement accordé) - comme l'a exercé la commission de réconciliation en Afrique du Sud aux coupables du système d'apartheid en cas d'aveu -, mais au contraire : il serait un droit des victimes de réclamer pour eux-mêmes le droit de traiter le coupable, qui a été jugé par le droit, au-delà du droit. Pour suspendre la puissance du droit pour un instant dans le droit même.

Pour illustrer cette absurde version d'un droit au-delà du droit dans le droit même qui a la force de suspendre la puissance du droit au cœur du droit,

24 Voir : Benjamin : Über den Begriff..., op.cit., p. 697 ; Sur le concept..., op.cit., p. 433. 
permettez-moi de vous donner à la fin de mon discours un exemple : l'exemple célèbre de Michael Kohlhaas de Heinrich von Kleist. ${ }^{25}$ Michael Kohlhaas - une figure réelle de l'histoire - a été vendeur de chevaux avec un sens de la justice (Rechtsgefühl: plutôt un sens du droit) ressemblant à un trébuchet; en bref, Kohlhaas est introduit par Kleist comme l'idéal du bon citoyen. Ce maquignon subit tout au début du récit une injustice : un suzerain, le chevalier von Tronka, réclame à tort de Kohlhaas des droits de douane. Kohlhaas est forcé de laisser deux chevaux en gage à von Tronka, que le chevalier ruine pendant les semaines à venir. Trois fois Kohlhaas porte plainte contre le chevalier à trois institutions judiciaires différentes trois fois le chevalier avec ses bonnes relations aux responsables des tribunaux réussit à rejeter les plaintes de Kohlhaas. Or, le droit se révèle comme pure instrument de puissance qui institue du droit là où il prétend se contenter de servir la justice (tout comme Benjamin l'a décrit dans Critique de la violence). Après ce triple échec, Kohlhaas fait ses adieux à tout système juridique et décide de prendre le chemin de vengeance. Or, il quitte la forme de justice appelée « droit » et se met à la forme de justice appelée vengeance ${ }^{26}:$ avec une foule en constante augmentation il circule dans tout le pays en chassant ses adversaires et en laissant des traces de destruction terrible et terrifiante partout. Cependant, la vengeance, elle aussi, n'atteint pas son objectif. Après une conversation longue et sincère avec Martin Luther, Kohlhaas décide de retourner dans le système juridique établit, pour que la faute du chevalier von Tronka puisse y être finalement jugée. Sous condition, bien entendu, que cette fois le droit ne se révèle plus comme le pur instrument du pouvoir pour protéger les puissants mais comme vrai moyen de la justice. Au moment où Kohlhaas réalise que - encore une fois - cela ne sera pas le cas, il a le choix de quitter encore une fois le système juridique et de continuer sa vengeance (parce qu'il obtient la possibilité de s'enfuir de prison). Ou de se soumettre à la puissance absolue du droit - sous condition que le chevalier von Tronka, lui aussi, soit sacrifié pour la conservation de la puissance du système. Kohlhaas, fatigué et seul, se prononce en faveur de l'option $\mathrm{n}^{\mathrm{o}} 2$, sachant que sa propre mort sera le prix. Autrement dit, Kohlhaas se prononce en faveur de la vengeance sous forme du droit. De cette manière le conte Michael Kohlhaas de Heinrich von Kleist se présente comme une illustration parfaite de la théorie du droit élaborée par Benjamin dans la Critique de la violence. En montrant également les conséquences révolutionnaires, c'est-à-dire de la destruction complète du droit : le cercle d'envoutement de la vengeance, la pure confrontation de la violence et d'une contre-violence avec des effets destructifs terribles.

25 Voir : Von Kleist, Heinrich : Michael Kohlhass et autres nouvelles, Phebus, Paris 1991.

26 Pour l'idée de la vengeance comme forme de justice voir : Christoph, Menke : Recht und Gewalt, August Verlag, Berlin 2012. 
Mais ce n'est pas, heureusement, la fin des possibilités. Tout au début du récit, au moment où Kohlhaas décide de quitter l'espace juridique pour se venger, sa femme, qui à ce moment est en train de mourir (à cause du chevalier von Tronka), signale à Kohlhaas qu'elle est d'accord avec sa décision de quitter le droit - mais justement pas pour se venger, mais pour pardonner le chevalier.

A ce moment-là, néanmoins, le pardon ne semble pas vraiment une alternative, parce qu'il aurait eu le caractère d'une décision purement privée (motivé par la religiosité de la femme de Kohlhaas) - sans le moindre effet politique. Le droit comme institution injuste ne serait pas touché par cette décision, le pardon serait un acte moral au-delà du droit. Cependant, la situation est autre à la fin du récit, au moment où le cas de Kohlhaas et du chevalier von Tronka est jugé par le tribunal final. A cet instant ni Kohlhaas ni von Tronka n'ont de pouvoir, personne n'a le droit d'agir, ils ont tous les deux pris par l'envoutement du droit ; l'alternative morale n'existe plus (au moins pas comme acte social). Il en serait autrement s'il y avait pour Kohlhaas un droit non-souverain à pardonner. Dans ce cas-là Kohlhaas - la victime, l'impuissant - aurait la possibilité de créer un véritable état d'exception, de créer un espace de non-droit dans le droit même - au lieu du souverain. Avec cela, le droit tout court n'est pas abolit ; mais la souveraineté fondant ou instituant le droit ne pourrait plus réclamer est souverain celui qui décide l'état d'exception, car il y a une deuxième instance qui a le même droit. Plus faible que le droit souverain, bien entendu, restreintes à certaines situations ou constellations. Mais quand même existantes, interrompant de cette manière la puissance absolue du droit. Comme le droit du coupable de se taire ou autres droits à inventer. Entsetzung des Rechts n'y est plus un seul acte révolutionnaire d'abolition complète du droit mais ce sont des moments - dans le sens hégélien du terme - d'interruption, d'arrêt, de mise entre parenthèses de la puissance du droit, de sa puissance d'instituer qui le rend identique à la puissance mythique. J'avoue que ces moments sont des moments très faibles, toujours à renouveler, ils ne présentent pas une fois pour toute la réponse au problème du côté mythique du droit, de sa violence spécifique. Mais ils peuvent - peut-être - aider à destituer le droit ici ou là, de temps en temps - en gardant de cette manière les traits du droit qui valent - encore une fois : peut-être - la peine d'être gardés.

Tobias Nikolaus Klass est, depuis 2007, maitre de conférences [Juniorprofessor, Akademischer Rat, Akademischer Oberrat] au Philosophisches Seminar de la Bergischen Universität Wuppertal, en Allemagne. Il a obtenu son Doctorat en Philosophie, intitulé « Das Versprechen. Zur Rhetorik sozialer Verbindlichkeiten im Anschluß an Searle, Hume und Nietzsche ", à l'Université de Bochum, en 2000. 